\title{
SERVICE DESIGN WITH MACHINE LEARNING BASED ON USER ACTION HISTORY
}

\author{
Xinyue WANG, Nobutada FUJII, Toshiya KAIHARA, Daisuke KOKURYO \\ Department of System Science, Graduate School of System Informatics, Kobe University, 1-1 Rokkodai-cho, Nada-ku, Kobe, Japan, \\ E-mail: wang@kaede.cs.kobe-u.ac.jp
}

\begin{abstract}
With the development of IoT techniques, it become easier to collect users' action data. By analyzing and using those data, consumers and producers will mutually exchange their intelligence and better customize product development processes. This study focuses on the users' daily life, examines a proposed system using sensor shoes with several sensor devices embedded in the insoles, collecting action data of users, extracting their action features, and then issuing some advice to help users train more efficiently. As described herein, a service model uses a backpropagation (BP) network to distinguish users' actions and to extract their action features using Self-organization Map from the presented sensing data. Experiments to confirm the feasibility of the proposed methods are undertaken. With the former result indicating an overall accuracy of $89.61 \%$ in distinction of 5 actions: sit, stand, walk, run and jump. The latter results showing that SOM is helpful to classify action feature in detail. After the analysing parameters of each cluster numbers, it is possible to make the action feature visualized by providing a colored cluster map, which makes it easier to compare train periods. Results also show the potential of utilization of data collected by devices to provide personal service.
\end{abstract}

Keywords: Neural network, Self-organizing map, Service Design

\section{INTRODUCTION}

Along with the progression of IoT (Internet of Things) technology studies, an increasing number of connected devices developed in a larger resources space, it is possible to improve human intelligence, which can also help in improving capabilities [1]. Concurrently, richer lives require an abundance of products and services. With the popularization of information and communication technology (ICT), it has become much easier to obtain more and more various information than ever before.

As one of the beneficiaries of these progression, consumers tend to choose products or services that are suitable for themselves more freely than before [2], which can raise the question of how to produce the most suitable products or services for consumers with different lifestyles. To ascertain consumers' changing needs, accumulating consumers' historical data showing their motivations and then exploiting needs from the historical data and providing services are expected to be necessary. In service engineering domain, from studies with themes such as individual services, system design for service model construction, sports science and health sciences have gradually gained in value from 2000[3]-[4].

Return to the topic of communication among the human, devices and internet. In recent years, development of IoT technology makes it possible to acquire users' personal data. The phrase "Internet of Things," (IoT) was first coined by Kevin Ashton in 1999 in the context of supply chain management [5]. Subsequently with the popularization of cloud computing technology is used with sensor devices [6]. Earlier, researchers had been aware of the concept of "connecting thing together". Mark Weiser also presented a smart environment of people's daily life through this concept [7]. Computers have become much more widely used since then, and have become useful anywhere and anytime. Embedding computer technology seamlessly in daily life, defined as ubiquitous computing, was undertaken and examined in various studies simultaneously. As a result, the number of connected devices exceeded the world population in 2011[8]. Although the definition of "things" has changed with the development of technology, the target of developing a computer that is independent from human beings with the capability of sense information and decision-making function will not change.

Beyond that, one of the implications of IoT system implementation is the changing role of customers, which becoming more active and connected, customers can now generate critical information about their behaviour and choices, which can be recorded in real time [9]. To customize products using personal data collected by IoT devices, product development has become a co-creation process involving producers and consumers.

Regarding studies of IoT technology utilization, anthropometric measurements - used in sports medicine and health science domains-have become used increasingly for application including the development of lives [10]-[11]. Applied researches with the aim that monitoring of human movement [12]-[13] or classifying of human movement for ambulatory monitoring [14]. Such researches were implemented by the utilization of IoT devices and obtained high accuracy in movement distinction or classification, which also showing the potential in public health care service under the background of global aging population. However, further applications of these results are expected, for instance, the wellbeing or supporting for the general population through their daily life.

Studies relating to the wellbeing or expansion of life, the Haptic Design project [15], with the theme of "body experience in the first person", specifically examined improvement of user happiness by sharing or expanding tactus information, or by designing an immersive environment for consumers [16]. Such studies devote attention to producing much better product experiences, but a great deal of discussions arises about the related data Because IoT devices can also collect data of different kinds, a more important task is how to process data correctly and efficiently [17]. Collecting as much data as 
possible is important, but little or no improvement can come from merely collecting a huge amount of data without analyzing or using it.

In the light of such findings, this study specifically examines design of a support service for daily life with consideration of utilization of the sensor data collected from a user.

A pair of sensor shoes [18] is used to collect a user's daily data. As mentioned above, many types of wearable sensors are utilized for collect user's action data. However, in most situations, a user's motivation is restricted in some degree by the position of sensors. The most common position is the waist using a triaxial accelerometer or others acceleration signal [14][19][20]. Since shoes are necessities of life, they can accumulate user action data easily. Moreover, it is possible for a user to spend daily life unconscious of sensors embedded in an insole. Such sensors are likely to reveal a user's demands and difficulties that a user might not be aware of in daily life. Embedded in an insole of a shoe could be an acceleration sensor, an angular velocity sensor, a geomagnetism sensor, and pressure sensors at three positions of the foot, along with a battery. Every sensor connects with a smartphone and sends sensor information by Bluetooth. Through the application algorithm installed in the smartphone, the user's acceleration, angular velocity, pressure at three positions, and other parameters are calculated and are then shown in the interface.

This study is unique. At the first, it explores about the using of sensing data collected by IoT devices and makes the data participate in the service design instead of only collecting and displaying. Secondly, this study starts from a new perspective which has few previous published literatures illustrates about the application of anthropometric measurements in the support of public daily life. In addition, the employment of the sensor shoes is also a new attempt. With the advantages described in the previous, the outcome is eagerly anticipated.

The paper is organized as follows: the next section explains objective and proposed service. In Section III, methods to implement proposed model are presented. In Section VI, computational experiments are described and results are demonstrated. Finally, conclusions and future work are discussed.

\section{OBJECTIVE AND PROPOSAL}

\subsection{Objective}

This research is aimed at the design of a service that gives advice for training by distinguishing a user's actions and extracting the actions features based on the user's action history data collected by sensor shoes.

\subsection{Proposed service}

Considering a service that records and collects action history data, a user who trains with sensor shoes has action features and difficulties that can be improved by comparison to previously set goal data. A user can then train much more efficiently at places not restricted to a gym. This report specifically describes training support service implementation.
A schematic diagram of the system is depicted in Fig. 1. Acceleration data, geomagnetism data, pressure data and other parameters are sent to the smartphone through Bluetooth in real time since a user wear the sensor shoe, and are saved in phone storage. A local computer is used to receive and analyze these sensor data, after the data mining, the action feature of user and some advices of target action are expected to be output and shown to user.

Because the movement state is not the same every time a user trains, recording various movement states is important for further analysis. The flow of the proposed service is the following.

- Goal setting: A user trains with a coach, records the sensing data, visualizes the action features by data mining, then sets a goal.

- Training: A user trains without a coach, accumulates sensing data, then extracts and visualizes the action features by data mining.

- $\quad$ Advising: The system compares results of training scores and advises a user to achieve the goal data.

- Goal data updating: A user who achieves the goal trains again with coach to update the goal data.

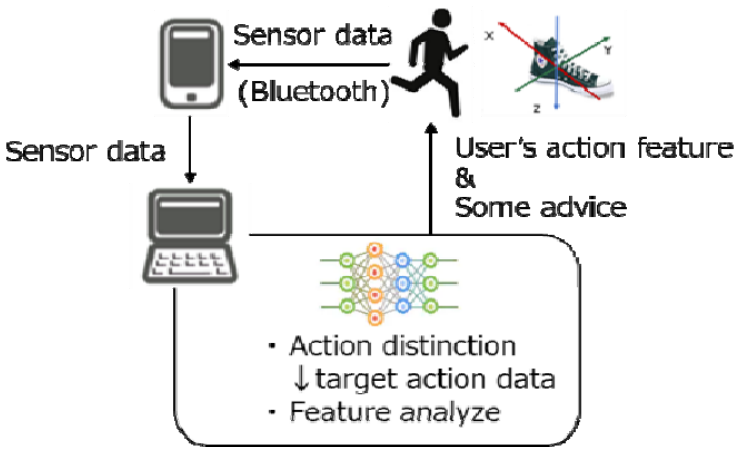

Fig. 1 Schematic diagram of the system

\subsection{Proposed model}

A model of training support service conducted in the local computer is depicted in Fig. 2, which consists of a calibration phase and training phase.

- $\quad$ Step 1: Calibration phase. Record various action data of a user as training data and train a network for distinguishing actions.

- $\quad$ Step 2-1: Training phase. Train with coach to set the goal data. A user trains according to the coach's instructions. Use the trained network in Step 1 to extract the target action data collected by sensor shoes from sensing data of each training bout. Use machine learning for data mining. Extract action features. Visualize goal data.

- $\quad$ Step 2-2: Train without coach while providing services. In this step, a user trains without a coach. Accumulate action data of each training bout and visualize action features. Compare the action features of each training bout and provide advice to approach the goal efficiently. 


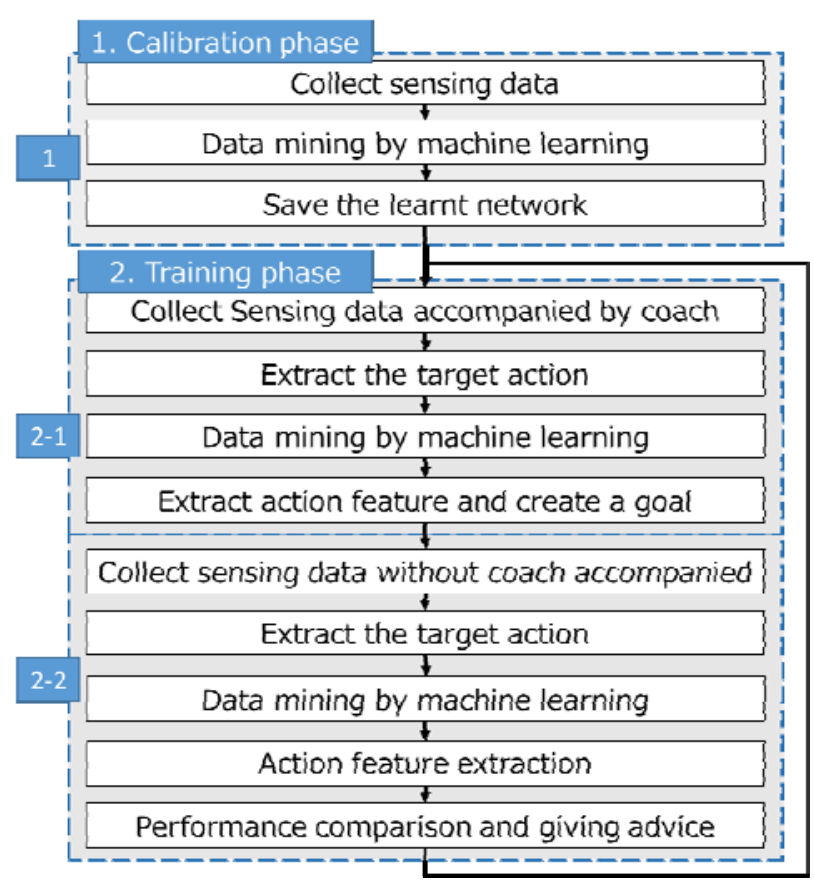

Fig. 2 Proposed model

\section{METHODS}

Machine learning will be used to implement data mining. In machine learning, sample datasets are input from sensors, databases, etc. and are analyzed. From those data, useful rules, knowledge representation, judgment criteria are extracted to develop algorithms [21]. Regarding studies also show that machine learning plays a powerful role in movement classifying and even pattern recognition such as Support Vector Machine $(\mathrm{SVM})[14][22]$ and Long Short-Term Memory (LSTM)[23].

Considering that the proposed model has a characteristic by which the action varies from person to person, distinguishing the actions of individual users correctly is necessary. This research uses supervised learning at the calibration phase. A user performs actions that may be done in training with the consciousness of "collect correct training data" to collect training data for training the network. In addition, as support for training, it is important to classify details of a user's sensor data, analyze them, and record action features. Therefore, this research uses unsupervised learning for feature extraction in step 2. The network automatically records and learns various action features when the user trains.

\subsection{Action distinction using Neural Network}

In step 1 , this paper specifically examines jogging training. Sensing data of actions of five types will be collected for standing, sitting, walking, jumping and running. Then supervised learning will be used to train the network. Subsequently, the actions of new sensing data are distinguished based on the trained network. Target action data are extracted and used for the next step.

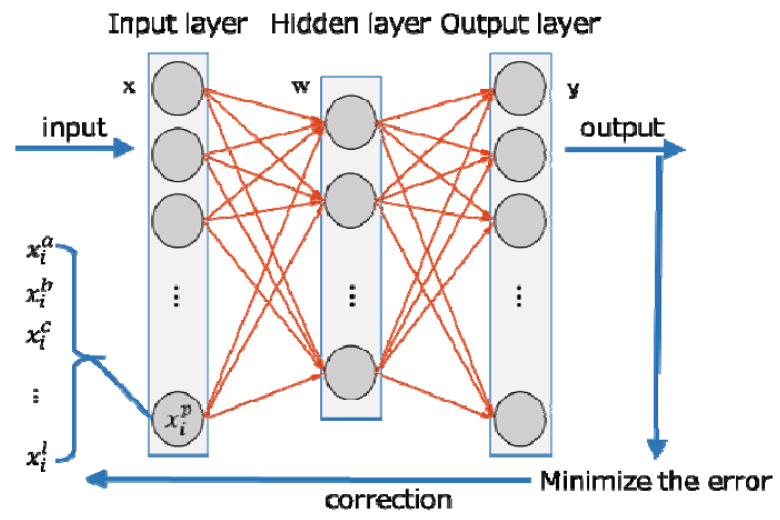

Fig. 3 Network with BP algorithm

A neural network with a backpropagation (BP) algorithm will be used to distinguish actions. BP is a neural network algorithm that corrects weights and biases by comparing training data and output [24]-[25]. As Fig. 3 shows, the network has an input layer, a hidden layer and an output layer. Neurons exist in each layer. The entire network is configured by connecting neurons. Weight $w$ is calculated based on the importance of the input signal and that of the error signal. Then it is updated in the hidden layer.

Common BP algorithm can be described as shown below.

A) Initial setting of each parameter.

B) Input training data.

C) Calculate the values of nodes in the hidden layer and output layer.

D) Verify the gradient of the error to judge if learning has ended. If the condition is satisfied, then the value of the weight vector is determined and learning is ended. Otherwise the parameters are updated by backward propagation.

E) Calculate the error value

F) Minimize the value of error by gradient descent

$\mathrm{G}$ Update the values of weight and bias; return to (C).

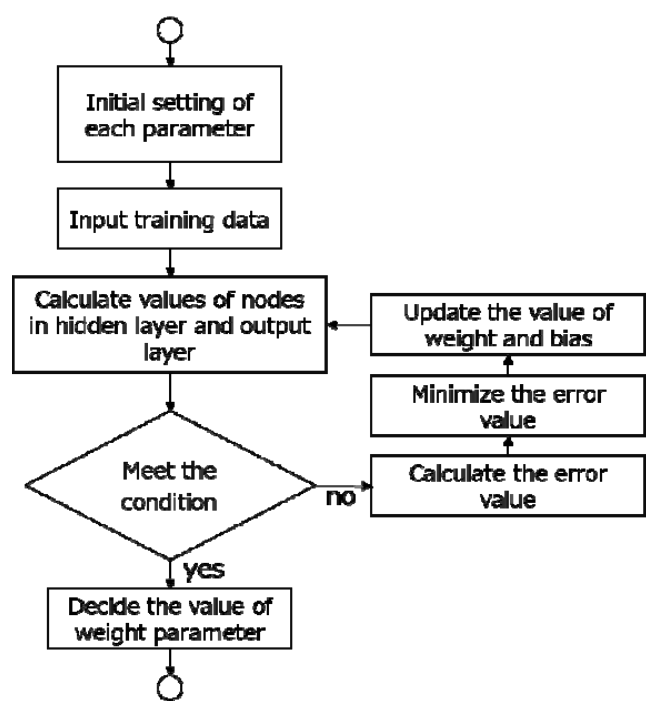

Fig. 4 BP algorithm 
In the input layer, 10-dimensional data are used for each neuron:

$$
x_{t}^{p}(p=\mathrm{a}, b, c, \ldots, h, k, l)
$$

$t$ : point in time

$a: \quad$ left/right foot

$b, c, d: \quad$ acceleration axis $\mathrm{x}-\mathrm{z}$.

$e, f, g: \quad$ angular velocity axis $\mathrm{x}-\mathrm{z}$.

$h, k, l: \quad$ pressures of heel, big toe, and little toe positions.

Weights between the input layer, hidden layer, and output layer are defined as $w_{i j}, w_{j k}$. Outputs of the hidden layer and output layer are defined as $H_{j}$ and $O_{k}$.

In the output layer, output is defined as shown below.

$$
\begin{aligned}
& Y_{i}=(\text { stand })\left[\begin{array}{lllll}
1 & 0 & 0 & 0 & 0
\end{array}\right]
\end{aligned}
$$

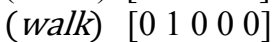

$$
\begin{aligned}
& \text { (run) } \quad\left[\begin{array}{lllll}
0 & 0 & 1 & 0 & 0
\end{array}\right] \\
& \text { (sit) } \quad\left[\begin{array}{lllll}
0 & 0 & 0 & 1 & 0
\end{array}\right] \\
& \text { (jump) [ }\left[\begin{array}{lllll}
0 & 0 & 0 & 0 & 1
\end{array}\right]
\end{aligned}
$$

A sigmoid function is used as an activation function:

$$
\mathrm{g}(\mathrm{x})=\frac{1}{1+e^{-x}}
$$

Error is calculated as

$E=\frac{1}{2} \sum_{k=1}^{m}\left(Y_{k}-O_{k}\right)^{2}$

where $Y_{k}$ and $O_{k}$ respectively denote the expected result and output. Gradient descent is used to make $E$ least.

\subsection{Feature extraction using self-organizing map}

Self-organizing Map (SOM) will be used for feature extraction to implement goal data setting and advising steps. SOM is a kind of unsupervised learning neural network proposed by Teuvo Kohonen [26] consisting of two layers: an input layer and an output layer. Input layer $N_{\text {in }}$ has $n$-dimensional numerical data. Output layer $N_{\text {out }}$ has nodes arranged in $n$-dimensional space. A weight vector $w_{n m}$ is attached to each node. SOM is trained by updating weight vectors. Fig. 5 depicts the construction of a common SOM network.

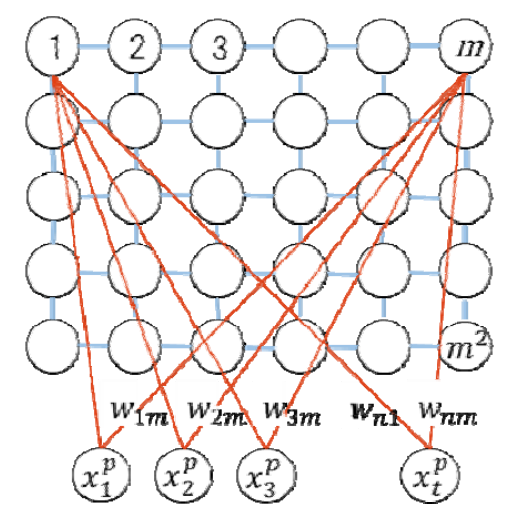

Fig. 5 Self-Organizing Map

Because the dimensions of the node arrangement of the output layer can be set freely, one can visualize the relation between the data by arranging the nodes in low dimensions and learning complex high dimensional data.

In the input layer, after a sample is selected randomly, its weight and distance between every neuron in the input layer are calculated. The most similar neuron can be chosen as a winner node. Subsequently neuron weights around the winner node will be updated. As described in

this paper, a 10 -dimensional data $x_{t}^{p}$ as section III-A are also used for each neuron in the input layer.

The algorithm can be described as explained below:

A) Initial setting of each parameter.

B) Input sample data $x_{t}^{p}$.

C) Set the neighbour radius $N_{j *}$.

D) Decide the winner node $j *$

E) Update the weights.

F) If learning coefficient $\eta$ arrive the set value, then finish the process; otherwise return to (C).

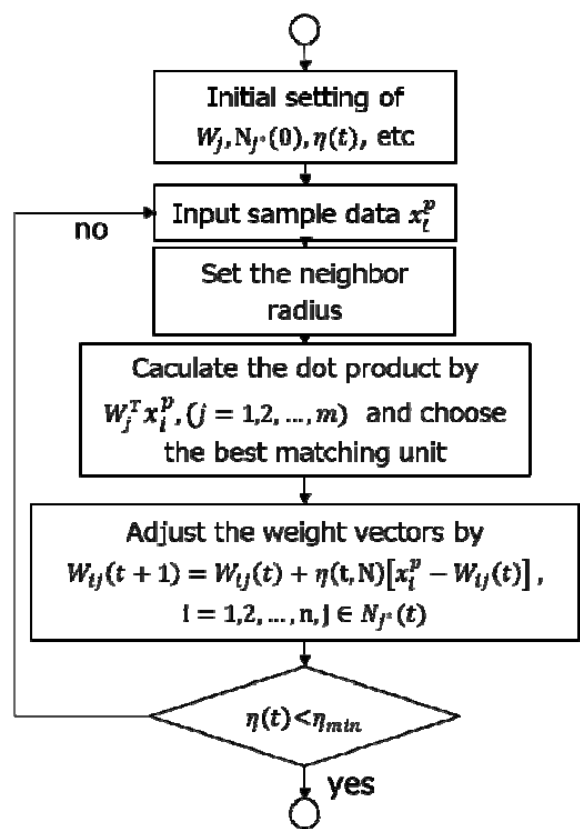

Fig. 6 Algorithm of SOM

\section{EXPERIMENTS}

\subsection{Action distinction}

In this section, an experiment is conducted to consider the accuracy of the action distinction method using a BP algorithm. The experimental condition is the following.

The user performs sitting, standing, walking, running, and jumping actions for $2 \mathrm{~min}$ each to collect sensing data. The measurement frequency of sensors is $10 \mathrm{~Hz}$, data of both feet are integrated. 2400 sensing data are collected for each action. Then 500 samples are extracted continuously from them randomly. Data extracted in each action are combined to form a matrix comprising 2500 columns and 10 rows.

Since each parameter of action has a period, in this experiment, training data was created by the integration of sensor data in $0.1 \mathrm{~s}, 0.5 \mathrm{~s}$ and $1.0 \mathrm{~s}$ period, with size of each matrix was 2500 columns and 20 rows, 50 columns and 100 rows, 25 columns and 200 rows. 
End condition of learning by validation verification: As described in this paper, if the gradient of the error does not change after six consecutive iterations, then learning is terminated to suppress over-learning.

Meanwhile, variable hidden layer node number was used to training the network. Number of hidden layer node and the average accuracy of training data in 3 period when the test accuracy was reaching convergence is shown in Table 1.

In the result table, the best average test accuracy is $89.62 \%$ with the period $0.1 \mathrm{~s}$, which also had the smoothest convergence curve showed in Fig. 7. Although the training data in period of $0.5 \mathrm{~s}$ and $1.0 \mathrm{a}$ got higher training accuracy, the test of accuracy was less than satisfaction, especially the test accuracy of walk and run had large fluctuation from $37.5 \sim 100 \%$. Nevertheless, this experiment had limited training data, increasing the quantity of training data or set a much more fixed time series cycle are regarded as improving the accuracy.

\section{Table 1 Result of NN}

\begin{tabular}{|c|c|c|c|c|}
\hline period & $\begin{array}{c}\text { input } \\
\text { node }\end{array}$ & $\begin{array}{c}\text { hidden } \\
\text { node }\end{array}$ & $\begin{array}{c}\text { training } \\
\text { accuracy }\end{array}$ & $\begin{array}{c}\text { test } \\
\text { accuracy }\end{array}$ \\
\hline 0.1 & 20 & 55 & $94.07 \%$ & $89.62 \%$ \\
\hline 0.5 & 100 & 200 & $97.31 \%$ & $84.02 \%$ \\
\hline 1 & 200 & 280 & $100 \%$ & $74.23 \%$ \\
\hline
\end{tabular}

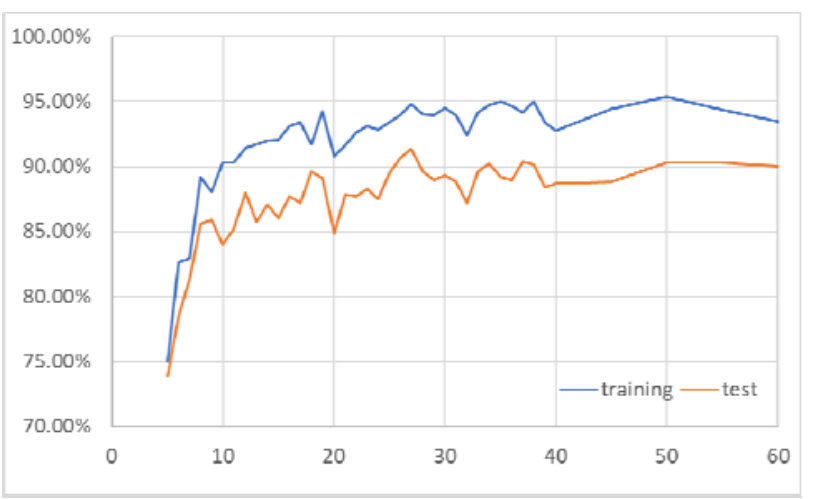

Fig. 7 Convergence curve of training data in period of $0.1 \mathrm{~s}$

\subsection{Feature extraction}

This section describes investigation of the effectiveness of the proposed method. Also, we conduct three experiments to assess action feature analysis by SOM. First, experiment 1 is designed to ascertain whether the user's action can be inferred from the cluster results. Experiment 2 is designed to investigate different states of the same action of one user. Finally, experiment 3 is designed to examine whether differences between the states of the same action of multiple users can be discriminated by cluster results. The conditions of the respective experiments are explained below.

- $\quad$ Experiment 1: The user wears sensor shoes and performs various actions to collect sensing data. About 3 min of walking action data are extracted as training data.
- Experiment 2: The user jogs about 2 min according to the instructions of a coach. Sensing data are collected and used as learning data (coach data). Subsequently, a user jogs about 2 min independently, collects sensing data, and uses the data as learning data (training data).

- $\quad$ Experiment 3: Two users jog the same distance. Collect sensing data and use it as learning data called data A and data B.

\section{1) Experiment for action state inference based on cluster results}

Results of clustering based on 2400 action data are obtained using Matlab SOM tools, as shown in Fig. 8. On the cluster map, the neurons are at the centre of each cluster. The area of the blue part indicates the number of data assigned to the respective clusters.

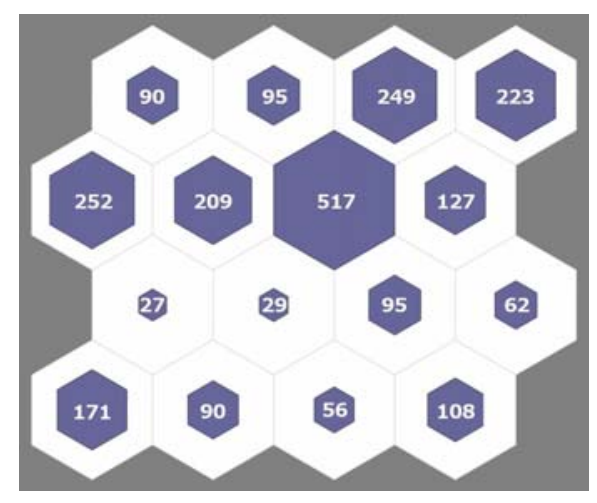

Fig. 8 Clustered data

Analyses of action features are based on mean values of each sensing data parameter. First, according to the pressure on the heel, the little toe and the big toe's position of each cluster's data, the user's action state is classified as a "foot is landing condition" or "foot is away from the ground condition".

\section{- Condition of landing}

Based on the magnitude of the pressure value at each position, the landing site is classified as "heel" or "toe". In addition, use of the $\mathrm{z}$ acceleration value can imply the weight of landing.

- Condition of foot above from the ground

In this condition, the pressure at each position is almost zero. Therefore, it is better to examine the acceleration specifically. According to the values of acceleration $\mathrm{x}$ and $\mathrm{y}$ axes, data are classified into three conditions: "putting down the foot", "lifting the foot" and "passing the zenith". Next, while referring to the z-axis acceleration values and the angular velocity, the momentum of walking and other detailed features can be inferred. The average value of each parameter calculated in each cluster is shown in Table 2: the so-called feature table.

In the feature table, if data of only one foot are classified into a cluster, then an L or R is appended after the cluster number in the first line. The state shown in the second line is described with 1 and 0 , signifying "condition of landing" and "condition of foot away from the ground". 


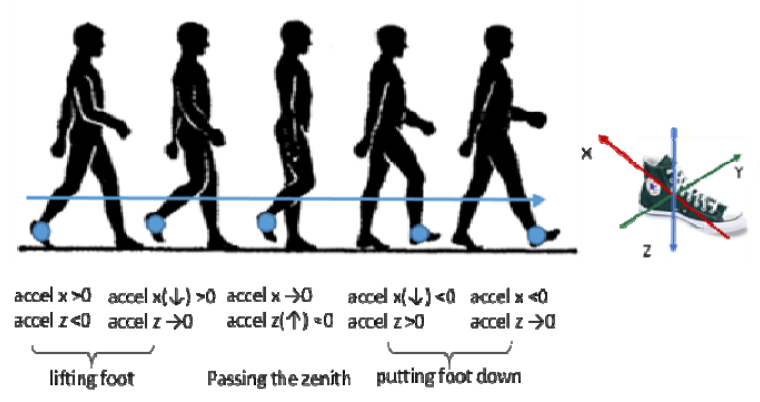

Fig. 9 Conditions of foot away from the ground

total number of data classified in a condition of landing. However, regarding the coach data, the data classified in the heel on the ground condition account for $10.15 \%$ of the total number of data classified in the condition of landing. Therefore, one can speculate that a user who trains according to a certain style will often land on the toes. When the foot is away from the ground, the pressure and $\mathrm{z}$ acceleration values are lager when a user trains with a coach. Therefore, if a user trains independently, the momentum of the action might be smaller. Therefore, some advice can be proposed: "Land by the heel more if you want to achieve the goal efficiently.

3) Experiment on comparing action states of multiple users

Table 2 Feature table

\begin{tabular}{|c|c|c|c|c|c|c|c|c|c|c|c|c|c|c|c|c|}
\hline $\begin{array}{l}\text { Cluster } \\
\text { parameter }\end{array}$ & 1 & 2 & 3 & 4 & $5 \mathbf{R}$ & 6 & 7 & 8 & 9R & $10 \mathrm{R}$ & 11 & 12 & $13 \mathrm{~L}$ & $14 \mathrm{~L}$ & 15 & 16 \\
\hline number & 171 & 90 & 56 & 108 & 27 & 29 & 95 & 62 & 252 & 209 & 517 & 127 & 90 & 95 & 249 & 223 \\
\hline condition & 1 & 1 & 0 & 0 & 1 & 0 & 0 & 0 & 1 & 1 & 1 & 0 & 1 & 1 & 0 & 0 \\
\hline $\operatorname{accel} x$ & L-0.77, R-1.97 & -0.48 & 41.59 & 2.84 & -0.15 & 33.51 & -12.53 & -82.74 & -0.56 & -0.15 & -1.02 & -22.79 & -0.84 & -0.18 & 8.37 & 5.11 \\
\hline accel y & L-1.91, R7.24 & 0.76 & L18.51, R-11 & 1.81 & 0.04 & -1.68 & 2.43 & 4.89 & 0.65 & 0.33 & 0.8 & 1.04 & 0.26 & 1.24 & -0.16 & 2.81 \\
\hline accel $z$ & 24.89 & 16.73 & 42.13 & 64.83 & 17.33 & -96.86 & 23.38 & 28.34 & 19.75 & 19.83 & 16 & 41.56 & 21.68 & 21.2 & 9.81 & 25.46 \\
\hline gyro $x$ & L-6.42, R2.62 & -0.27 & 1.85 & -5.18 & 0.26 & -11.07 & 1.14 & L-3.57, R7.72 & -0.89 & -0.11 & 0.63 & 3.72 & -1.2 & -2.73 & L10.02,R-5.85 & L10.58, R-6.78 \\
\hline gyro y & 20.44 & 1.88 & 64.07 & 62.6 & 0.89 & 33.17 & 33.49 & L-2.77, R-5.41 & 5.98 & 4.69 & 0.14 & -41.61 & 9.58 & 10.88 & -26.03 & 44.12 \\
\hline gyro z & L4.42, R-19.31 & -0.69 & L-30.5, R7.19 & -2.82 & -1.07 & -3.48 & -3.73 & L4.17, R-5.94 & -1.04 & -1.01 & -0.62 & 7.26 & -0.93 & -1.54 & L6.94, R0.54 & L4.64, R-2.98 \\
\hline heel & 104.61 & 54.32 & 0 & 0 & 60.7 & 9.93 & 0.38 & 0 & 0.21 & 1.69 & 1.33 & 0 & 0 & 0.32 & 0 & 0 \\
\hline little toe & 0 & 0 & 0 & 0 & 68.37 & 0 & 1.54 & 0 & 107.81 & 75.84 & 1.56 & 0 & 46.31 & 5.64 & 0 & 0 \\
\hline big toe & 0 & 0 & 0 & 0 & 1.52 & 0 & 1.96 & 0 & 42.08 & 9.63 & 3 & 0 & 99.89 & 75.15 & 0 & 0 \\
\hline
\end{tabular}

Based on the feature table, the coloring of cluster results is shown in Fig. 10. Yellow represents the landing condition. The darker cluster shows that the heel pressure is greater. The lighter cluster shows that the toe pressure is greater. Blue represents "putting down the foot". Also, lighter colour show smaller $\mathrm{x}$ direction acceleration. Purple shows "lifting of the foot", with lighter colours representing smaller $\mathrm{x}$ direction acceleration. The other conditions are shown in transparent colour. Features of walking can be investigated. Similar conditions are colored similarly based on the table. Results shows that clusters with similar conditions are assigned in the near place. Therefore, one can use SOM to analyse a user's action features.

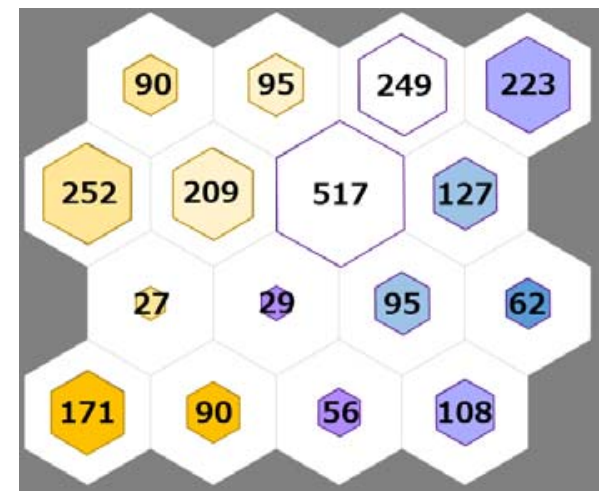

Fig. 10 Colored cluster result of Experiment 1

\section{2) Experiments to assess action states from one user}

The colored cluster results of 1412 training data and 1340 coach data using SOM are shown in Fig. 11. Regarding training data, the data classified as being in the heel on the ground condition account for $4.75 \%$ of the
The colored cluster results of 1371 data $\mathrm{A}$ and 1177 data B using SOM are shown in Fig. 12. Regarding the condition of the foot landing, it can be speculated that user A lands more often than user B because user A occupies $62.71 \%$ and user B occupies $81.44 \%$ of the total number of data. Furthermore, the ratio of the number of data for lifting the foot when the foot is away from the ground is $63.86 \%$ for $\mathrm{A}$ and $51.73 \%$ for $\mathrm{B}$. It can be speculated that user A takes more time for lifting of the foot than user B. Consequently, SOM can suggest different features of the same action of multiple users.

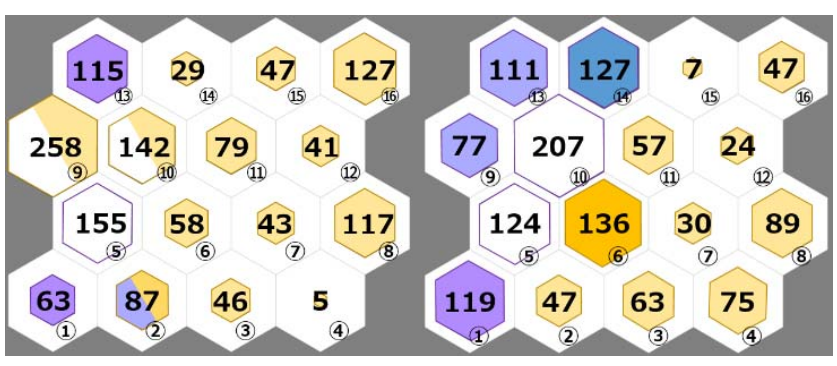

Fig. 11 Colored cluster results of coach data (left) and training data (right)

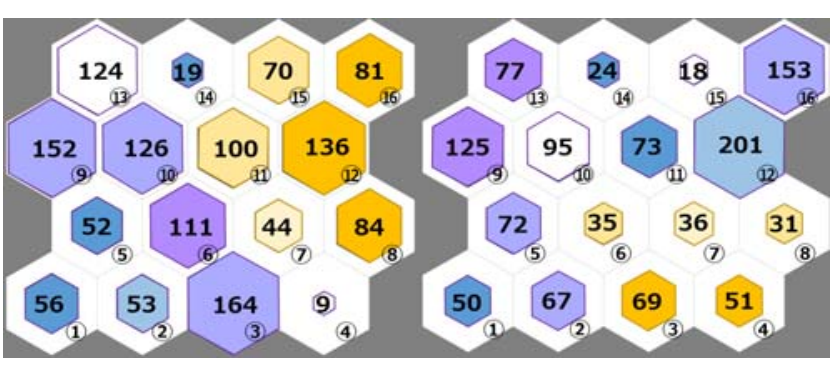

Fig. 12 Colored cluster results of data A (left) and data B (right) 
The result of experiments above indicates the possibility of using SOM to classify and infer the user's action. Since the cluster position is fluid in every learning result, the doubt that whether the results of clustering is reliable or not. A subsidiary experiment was conducted to investigate the reliability of the cluster position in each changing learning result.

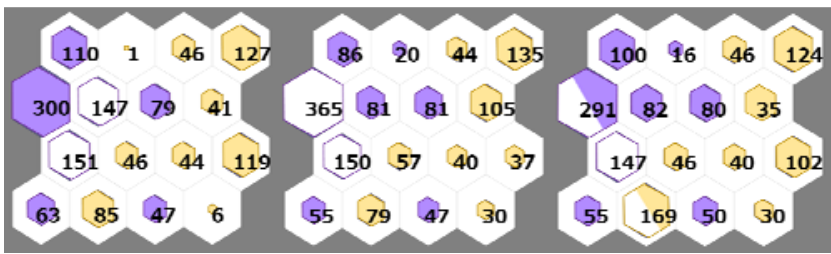

Fig. 13 Colored cluster results of training data in three times

Three colored cluster results of 1412 training data which was used in experiment 2) using SOM are shown in Fig. 13. As before, yellow represents the landing condition and purple shows "lifting of the foot". Regarding the position distribution of clusters in each result, it is obviously that every time has a similar distribution, even the adjacent clusters have not seemed to change much. Although there are slight changes in the number of data assigned to the respective clusters, the method is also feasible.

\section{CONCLUSIONS}

In action distinction, although one can distinguish actions by sensing data from one user using the BP algorithm, the accuracy is still unsatisfactory. Therefore, it is necessary to improve the data processing or algorithm itself. In feature analysis, one can infer a user's action state from cluster results obtained using SOM. Then one can investigate each cluster's feature. Moreover, one can compare different states of the same action of one user from extracted features, making it possible to propose advice to support efficient training. However, the advices proposed at present are manually judged, future studies should attempt to implement the advising by algorithm itself.

\section{REFERENCES}

[1] ROGERS, Y.: Moving on from Weiser's vision of clam computing: engaging ubicomp experiences. Ubiquitous Computing, 2006.

[2] NAITO, K.: Service Engineering, University of Tokyo Press, 2009.

[3] TAKESHI, T. et al.: Service Research Strategy toward Value Co-Creation, Information Processing Society of Japan, Vol. 50, pp. 1539-1548, 2008.

[4] MAGOME, T.: Development in Sports Analysis and Sports Medicine domain using IoT sensor Technology, Informatics Society Magazine, No. 46, pp. 112-116 2018 (translated from Japanese).

[5] ASHTON, K.: That "internet of things" things. RFid, Vol. 94, 2009.

[6] ITU. Internet of things global standard initiative. 2015.
[7] GOLD, R. et al.: The origins of ubiquitous computing research at PARC in the late 1980s. IBM Systems, 1999.

[8] BUYYA, R. et al.: Internet of things (IoT): A vision, architectural elements, and future directions. Future Generation Computer Systems, Vol. 29, pp. 1645$1660,2013$.

[9] SAYAR, D.: The antecedents of Successful IoT Service and System Design: Cases from the Manufacturing Industry. International Journal of Design, Vol. 12, No. 1, 2018

[10] IoTechNews, A wearable device improves your eating habits (translated from Japanese), iotechnews.com/2015/01/14/bitbite/.

[11] U-NOTE, An electric toothbrush with embedded Bluetooth.(translated from Japanese), u-note.me/note/47499535.

[12] SHANY, T. et al.: Sensors-Based Wearable Systems for Monitoring of Human Movement and Falls, IEEE Sensors Journal, Vol. 12, No. 3, pp. 658-670, 2012.

[13] SHARMA, A. et al.: High Accuracy Human Activity Monitoring using Neural Network, Third 2008 International Conference on Convergence and Hybrid Information Technology, pp. 430-435, 2008.

[14] KARANTONIS, D. M. et al.: Implementation of a Real-Time Human Movement classifier Using a Triaxial Accelerometer for Ambulatory Monitoring, IEEE Transaction on information technology in biomedicine, Vol. 10, No. 1, 2006.

[15] MINAMIZAWA, K.: Haptic design project, hapticdesign.org.

[16] YOKOYAMA, R.: An extraordinary experience produced by the combination of vision, auditory sense and tactus, hapticdesign.org/designer/file016_Ryo_Yokoyama.

[17] HASSANALIERAGE, M. et al.: Health monitoring and management using internet-of-things (IoT) sensing with could-based processing: Opportunities and challenges. IEEE International Conference on Services Computing, Vol.47, pp. 285-292, 2015.

[18] Fujitsu Limited-interactive shoe hub-android API. Fujitsu Confidential, 2016.

[19] MATHIE, M. J.: Monitoring and Interpreting Human Movement Patterns Using a Triaxial Accelerometer. Ph.D. Thesis, University of New South Wales, Kensington, Australia, 2003.

[20] TAMURA, T.: Classification of Acceleration Waveforms during Walking by Wavelet Transform, Methods of Information in Medicine, 36(04/05): 356-359, 1997.

[21] MITCHELL, T.: Machine Learning. McGraw-Hill, 1997.

[22] MARTISKAINEN, P. et al.: Cow behavior pattern recognition using a three-dimensional accelerometer and support vector machines, Applied Animal Behavior Science 119, pp. 32-38, 2009. 
[23] PENG, Y. et al.: Classification of multiple cattle behavior patterns using a recurrent neural network with long short-term memory and inertial measurement units, Computers and Electronics in Agriculture 157, pp. 247-253, 2019.

[24] FLACH, P.: Machine learning-The art and science of algorithms that make sense of data-. 1997.

[25] IAN, G. et al.: Deep learning. MIT Press, 2016.

[26] KOHONEN, T.: Self-Organizing Maps. Springer Series in Information Sciences, 2001.

Received March 2, 2020, accepted June 6, 2020

\section{BIOGRAPHIES}

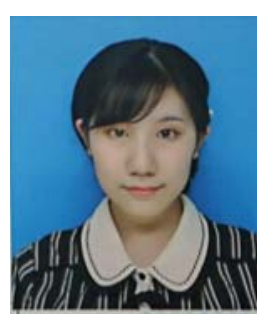

Xinyue Wang Received the Bachelor of Engineering degree in computer science and engineering from the School of Optical-Electrical and Computer Engineering, University of Shanghai for Science and Technology, China, in 2016 and the Master of Systems Science degree from the Graduate School of System Informatics, Kobe University, Japan in 2019, where she is currently pursuing the Ph.D. degree in Engineering. Her current research interests include the service engineering, Internet of Things, and machine learning.

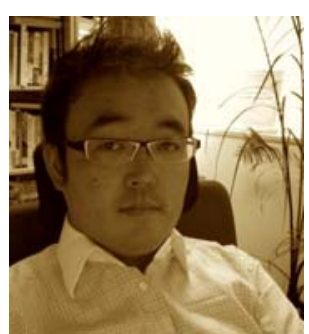

Nobutada Fujii. Received the Master of Engineering degree from the Kobe University, and the Ph.D. degree in Engineering from Tokyo University. $\mathrm{He}$ is currently an Associate Professor at the Graduate School of System Informatics, Kobe University, Japan. His research work has covered areas ranging from selfcontrolled and distributed production system, service engineering. N. Fujii was the Advances in Production management Systems special session organizer for conferences (2013, 2014 and 2015). He is a number of The Japan Society for Precision Engineering, The Japan Society of Mechanical Engineers, and the program committee of Japan Society for Serviceology.

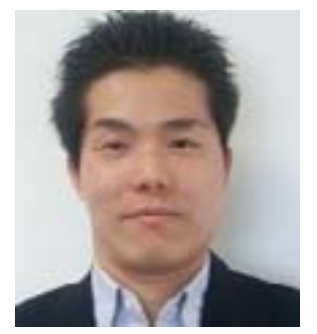

Daisuke Kokuryo. Received the Master of Engineering degree from the Kobe University, where received the Ph.D. degree in Engineering. $\mathrm{He}$ is currently an assistant professor at the Graduate School of System Informatics, Kobe University, Japan. His research works has covered areas from Medical systems, Biomaterials and Biomedical engineering. He is a number of the Japan Society of computer Aided Surgery, Japanese Society for magnetic resonance in medicine, and the Institute of Systems.

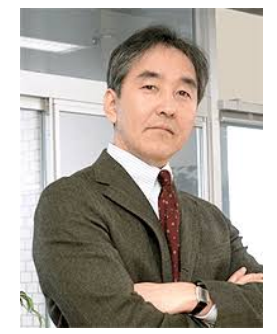

Toshiya Kaihara. Received the Master of Engineering degree from the Kyoto University, and the Ph.D. degree in Engineering from Imperial College London. He is currently a Professor and vice dean at the Graduate School of System Informatics, Kobe University, Japan. He has been involved with research in multi-agent system which is applied to production, service system and social system, management information and service engineering. He has authored 250+ refereed journals, conference proceedings, and book chapters. He is the president of the institute of Systems, Control and Information Engineers, the manager of the production system department of The Japan Society of Mechanical Engineers, the director of Japan Society for Serviceoogy. 